\title{
EINFLUSS VON KOPIENZAHLVARIATIONEN AUF DIE TUMORENTWICKLUNG
}

\author{
Christoph Standfuß, Andreas Klein, Heike Pospisil
}

\section{Zusammenfassung}

Tumorentstehung ist ein Prozess, bei dem die Abläufe innerhalb der Zelle schrittweise verändert werden. Die vielfältigen Interaktionen bei der Tumorentstehung sind jedoch bislang nicht vollständig erforscht. Bisher wurden vorwiegend Genexpressionsanalysen genutzt, die jedoch nur eine Zeitaufnahme aller Genexpressionen innerhalb der Zelle darstellen und somit allein nicht ausreichend zur Charakterisierung eines Tumors. Wir haben mithilfe von Affymetrix Mouse Diversity Genotyping Microarrays Mausbrustdrüsengewebe entsprechend unserem Dreistufen-Mausmodell analysiert und die Kopienzahländerungen berechnet. Wir fanden eine zunehmende stufenweise Änderung von den transgenen zu den Tumorproben. Die Berechnung von chromosomalen Segmenten mit gleicher Kopienzahl zeigte deutliche Fragmentmuster.

Unsere Analysen zeigen, dass die Tumorentstehung ein schrittweiser Prozess ist, der sowohl durch Amplifikationen als auch Deletionen chromosomaler Abschnitte definiert ist. Wir fanden charakteristisch konservierte Fragmentierungsmuster und individuelle Unterschiede welche zur Tumorgenese beitragen.

\section{Abstract}

Tumor development is known to be a stepwise process involving various changes that affect cellular integrity and behavior. The complex interactions and relationships between genomic organization and gene, as well as protein expression are not yet fully understood. To date, research has mainly focused on gene expression profiling or alterations in oncogenes, but these results show only snapshot of the cell status and are not sufficient to describe the whole tumorigenesis process.

We analyzed DNA from mouse mammary gland epithelial cells according to a three stage mouse model using the Affymetrix Mouse Diversity Genotyping array, calculated the CNAs and found a stepwise increase in CNA during tumor development. The segmental copy number alteration revealed informative chromosomal fragmentation patterns.

Our analyses suggest genome reorganization as a stepwise process that involves amplifications and deletions of chromosomal regions. We conclude from distinctive fragmentation patterns that conserved as well as individual breakpoints exist which promote tumorigenesis.

\section{»I. EINLEITUNG}

Krebs zählt zu den häufigsten Todesursachen weltweit und entsteht in einem schrittweisen Prozess, der sowohl die zellulären Eigenschaften als auch das Verhalten innerhalb einer Zelle beeinflusst [Hanahan und Weinberg 2000]. So weiß man seit Jahrzenten, dass verschiedene Stoffwechselwege die Entwicklung von Tumoren begünstigen und initiieren. Neben Veränderungen einer einzelnen Zelle, bilden vor allem die Veränderung der Kommunikation zwischen Zellen, Veränderungen des Zellstoffwechsels und die veränderten Reaktionen des Immunsystems die Grundlage für eine schrittweise Entwicklung von Krebsgeschwüren [Klein et al. 2005, Hanahan und Weinberg 2000, Osborne et al. 2004].
Ziel unserer Studie war die Untersuchung der Entwicklungen und Auswirkungen von Kopienzahlvariationen bei der Tumorentwicklung in gesunden und transgenen Mausbrustdrüsen. Von Kopienzahlvariationen spricht man, wenn ein genomischer Abschnitt mehrfach oder weniger als normal ${ }^{1}$ vorkommt. Ursache hierfür ist die Duplikation oder Deletion chromosomaler Abschnitte. Um die Karzinogenese gezielt initiieren zu können, trugen die transgenen Tiere das WAP/SVT Hybridgen, das aus dem WAP Promotor (kodierend für das wichtigste Milchprotein in Säugetieren) und der kodierenden Region des SV40 Gens [Klein 2005] besteht. Wird die Laktation während der Schwangerschaft aktiviert, so kommt es zu einer anhaltenden Aktivierung von SV40, was in Folge zur Tumorbildung in allen Mäusen führt. Wir beschreiben dazu ein dreistufiges Mausmodell [Standfuß et al. 2012], welches aus Brustdrüsen von gesunden Mäusen, transgenen Mäusen ohne Tumor, transgenen Mäusen mit Tumor und transgenen Mauszelllinien besteht.

Unser Hauptfokus lag zum einen auf der Analyse von Kopienzahlveränderungen während der Tumorentstehung und zum anderen auf der Untersuchung eines Zusammenhanges zwischen genomischen Veränderungen und der Änderung der Genexpression. Ein weiteres Ziel war es, genomische Veränderungen zu erkennen, welche die Entwicklung von Tumoren begünstigen bzw. vorhersagen können. Dazu verwendeten wir Affymetrix Mouse Diversity Genotyping Microarrays, um Kopienzahlvariationen, und Affymetrix Expressionsarrays, um die Genexpression zu bestimmen. 


\section{» II. ERGEBNISSE}

Wir wissen heute aus jahrelanger Forschung und verschiedenen Studien, dass die Veränderung der Kopienzahlvariation ein wichtiger Aspekt bei der Krankheitsentstehung ist und die Mutation einzelner Gene nicht allein ausreicht [Bergamaschi et al. 2006]. Aus früheren Studien ist bekannt, dass die Veränderungen der Kopienzahl eine wichtige Rolle bei Krankheiten spielen [Fanciulli et al. 2010]. Andere Studien zeigen, dass cirka 3\% des Genoms eines gesunden Menschen leichte Veränderungen in der Kopienzahl aufweisen [Conrad et al. 2009, Redon et al. 2006]. Bei Mäusen schwankt diese Zahl zwischen 3\% [Cahan et al. 2009] und 10,7\% [Henrichsen et al. 2009].

Durch die Entwicklung vom Microarrays zur Analyse von Kopienzahlvariationen ist es möglich, diese Kopienzahlvariationen in verschiedensten Proben zu analysieren und miteinander zu vergleichen. So konnte eine Arbeitsgruppe um Juliane Hannemann und Burkhard Brandt mithilfe der Microarray-Technologie Veränderung von Kopienzahlvariationen innerhalb des EGFR Gens zeigen, ein wichtiges Gen zur Diagnose und Therapie von Mamakarzinomen [Hannemann 2012]. Die von uns verwendeten Microarrays eignen sich hervorragend für solche Analysen und basieren auf sogenannten SNP Proben. Diese Proben stellen die häufigsten über das Genom verteilten Variationen eines einzelnen Nukleotides dar. So lässt sich, trotz begrenzter Kapazität eines Microarrays, eine gute Abdeckung des Genomes erreichen. Anhand der Kopienzahlen dieser SNPs haben wir dann die Analysen genomischer Veränderungen durchgeführt.

Die Proben unserer gesunden Mäuse zeigen eine vergleichbare Veränderung der Kopienzahlen, wie sie bereits in der Literatur beschrieben wurde (Abb. 1). In unserer Studie konnten wir zeigen, dass eine klare Zunahme der Kopienzahlvariation während der Tumorentwicklung zu beobachten ist.

Um die Kopienzahlvariationen der chromosomalen Abschnitte genau zu untersuchen, haben wir Abschnitte mit gleicher Änderung der Kopienzahl benachbarter SNPs zu Segmenten zusammengefasst (Abb. 2). Vergleicht man diese Abbildung, so lässt sich bereits
Kopienzahländerungen in
normalem Gewebe
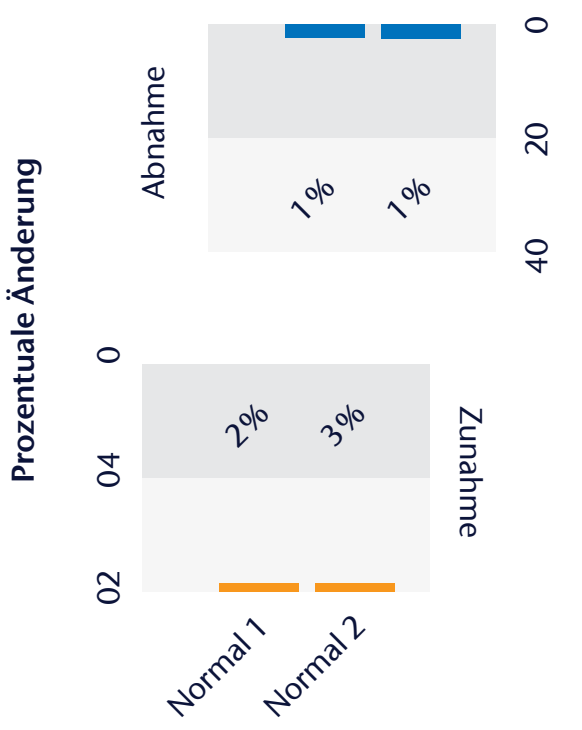

Abb. 1) Ermittelte Kopienzahlvariationen in beiden normalen Proben. Der Vergleich mit Werten aus der Literatur zeigt, dass beide Proben vergleichbar mit den von Cahan beschriebenen Werten sind [Cahan et al. 2009].

erkennen, dass in der normalen Probe keinerlei Segmentierung zu beobachten ist. In der transgenen Probe hingegen lassen bereits beginnende Änderungen chromosomaler Abschnitte erkennen, die in der gezeigten Tumorprobe weiterhin deutlich zunehmen. Vergleicht man die Segmentierung der Tumorprobe und der transgenen Probe, so lässt sich auch eine leichte Zunahme der Segmente erkennen.

Betrachtet man den prozentualen Anteil an genomischer Veränderungen, lassen sich diese Beobachtungen gut quantifizieren. Abbildung 3 zeigt diese prozentualen Kopienzahlveränderungen der errechneten Segmente, wobei diese zur Visualisierung in leichte Zunahme (orange) und leichte Abnahme (hellblau) sowie starke Zunahme (rot) und starke Abnahme (dunkelblau) gruppiert wurden. Während bei den normalen Gewebeproben nur 21\% der Segmente eine Veränderung der Kopienzahlvariation zeigen und diese in den transgenen Proben mit 26\%-28\% etwas höher ausfällt, sind fast die Hälfte aller errechneten Segmente in beiden Tumorproben deutlich verändert. Dabei lässt sich im Tumor 1 überwiegend eine Abnahme der Kopienzahl feststellen, was eine Deletion dieser Abschnitte in vielen Zellen der Probe bedeutet, während im Tumor 2 die Amplifikationen verschiedener
Bereiche überwiegen. Diese prozentualen Veränderungen zeigen somit eine deutliche Zunahme der Veränderungen bei der Tumorentstehung.

Um den Zusammenhang von Genexpression und Kopienzahl zu untersuchen, haben wir genomische Bereiche mit veränderter Kopienzahl und die Expression betroffener Gene miteinander verglichen. Beispielhaft dafür ist ein chromosomaler Abschnitt von Chromosom 6 (17.4 Mb-18.6 Mb) in Abbildung 4 dargestellt. In diesem Bereich lassen sich die 4 berechneten Segmente erkennen, für die eine bis zu 3-fache Zunahme der Kopienzahlvariation ermittelt wurde. Betrachtet man dazu die entsprechende Genexpression der in diesem Abschnitt lokalisierten Gene, lässt sich eine hochregulierte Genexpression in 4 betroffenen Genen feststellen. Lediglich 2 Gene, deren Kopienzahl ebenfalls hoch ist, zeigen einer erhöhte Genexpression. Es lässt sich jedoch feststellen, dass diese direkte Korrelation nur für wenige Beispiele gefunden werden konnte. In den meisten Fällen lässt sich jedoch keine Korrelation feststellen. Dies entspricht den Ergebnissen der Arbeitsgruppe von Sven Nelander, welche zeigen konnten, dass sich die komplexen Zusammenhänge von Kopienzahländerung und Genexpression nicht direkt, aber durch transkriptionelle Netzwerke modellieren lassen [Jörnsten et al. 2011].

Da der gezeigte Bereich der Kopienzahlvariation lediglich in einer Tumorprobe zu beobachten war, überprüften wir die gezeigten Veränderungen mittels einer quantitativen PCR. Die entsprechenden Primerpaare wurden dazu in einem Bereich ohne Kopienzahlveränderung vor Met (Met_ua) und an 2 Stellen des ersten Segmentes mit erhöhter Kopienzahlvariation (Met_am1, Met_am2) gelegt. Vergleicht man die Ergebnisse der qPCR in Abbildung 5 so ist zu erkennen, dass die Erhöhung der Kopienzahl bestätigt werden könnte.

\section{» III. AUSBLICK}

Wir konnten zeigen, dass die Tumorentwicklung mit einer schrittweisen Veränderung der genomischen Struktur und damit auch Kopienzahlveränderungen einhergehen. Der Vergleich der ermittelten Segmente in den verschiedenen 


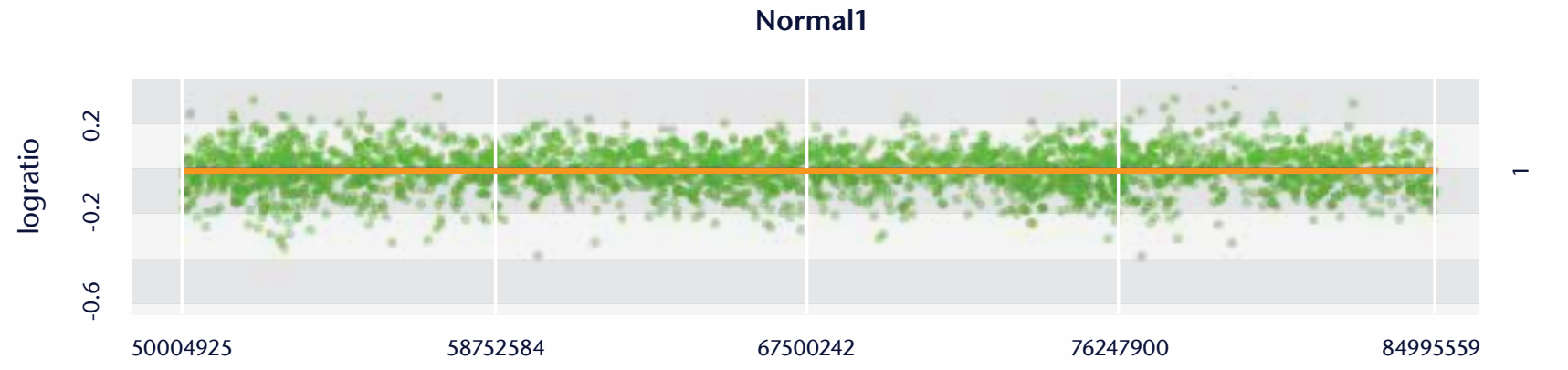

maplocation [bp]

Transgenic1

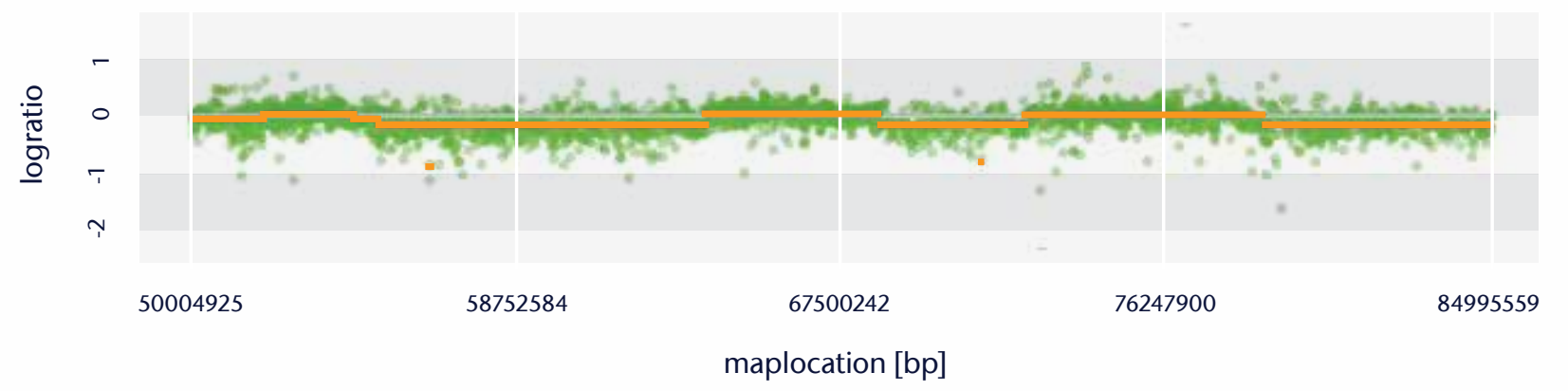

Tumor1

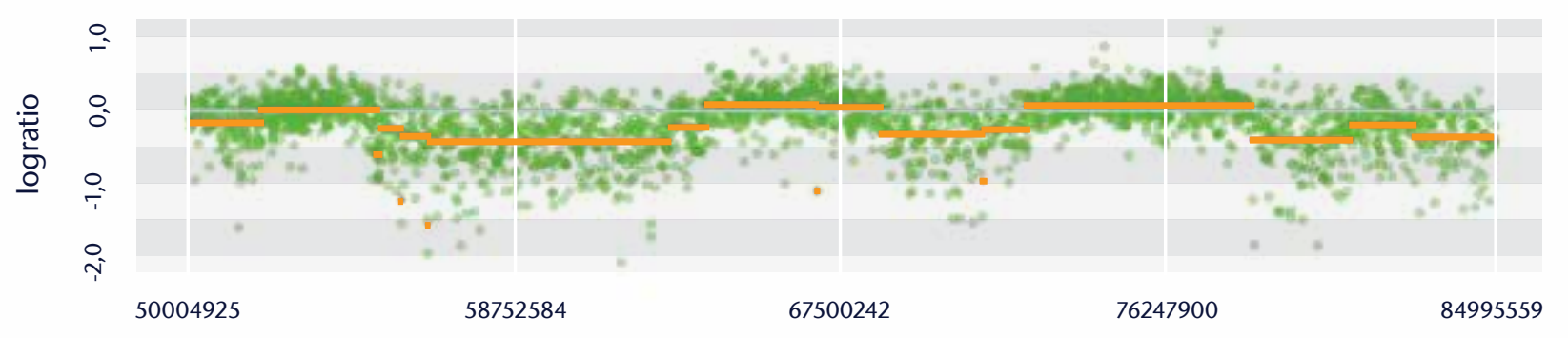

Abb. 2) In dieser Abbildung sind die Kopienzahländerungen der einzelnen SNPs (grün) und die daraus ermittelten Segmente gleicher Kopienzahl (dunkelrot) in 3 verschiedenen Geweben dargestellt. Im normalen Gewebe lassen sich keinerlei Änderungen in der Kopienzahl des errechneten Segmentes feststellen. In der transgenen Probe nimmt die Schwankung der SNP Kopienzahlen, sowie die Fragmentierung und die Kopienzahländerung der entsprechenden Segmente leicht zu. Vergleicht man diese Probe, mit der Tumorprobe erkennt man nicht nur eine Zunahme der Kopienzahländerung, sondern auch eine weitere Fragmentierung der vorherigen Segmente.

Proben zeigte hierbei einen schrittweisen Anstieg der Kopienzahlen von Normal über Transgen zu Tumor. Fast 50\% der Segmente wiesen dabei eine deutliche Änderung der Kopienzahl auf. Auch wenn in vielen Fällen keine direkte Korrelation von Kopienzahländerung und Genexpression zu ermitteln war, konnten wir einzelne Fälle ermitteln und aufzeigen. Gleichzeitig bestätigten wir die beobachteten Kopienzahlvariationen mittels einer qPCR. Bei vergleichender Betrachtung der Kopienzahländerungen in beiden Tumorproben lässt sich jedoch feststellen, dass jeder Tumor individuell ist und nicht jede Änderung in beiden Proben zu beobachten ist.

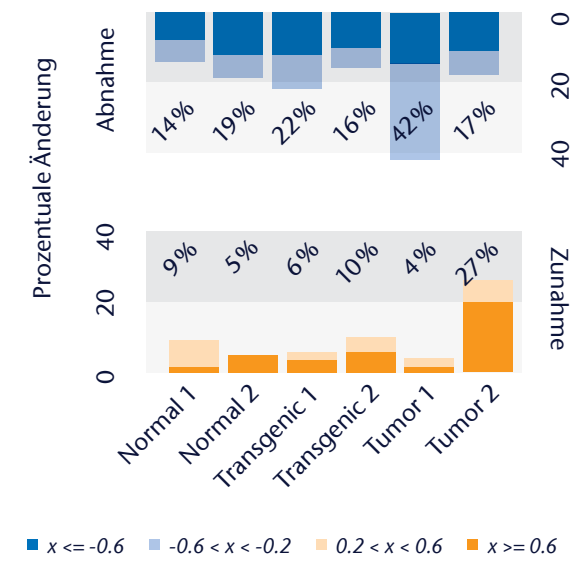

Abb. 3) Die prozentuale Änderung von Kopienzahlen der Segmente in den einzelnen Proben ist in dieser $A b$ bildung dargestellt. Vergleichend betrachtet lässt sich ein Anstieg der Kopienzahlvariation der Segmente im Tumor ( 46\%) gegenüber der transgenen ( 28\%) und normalen Probe ( 14\%) feststellen. Vergleicht man die beiden Tumorproben, so überwiegt im Tumor 1 der Anteil an Deletionen (Kopienzahlabnahme), während im Tumor 2 der größere Prozentsatz eine Amplifikation (Kopienzahlzunahme) darstellt. 


\section{Quantitative Realtime PCR}

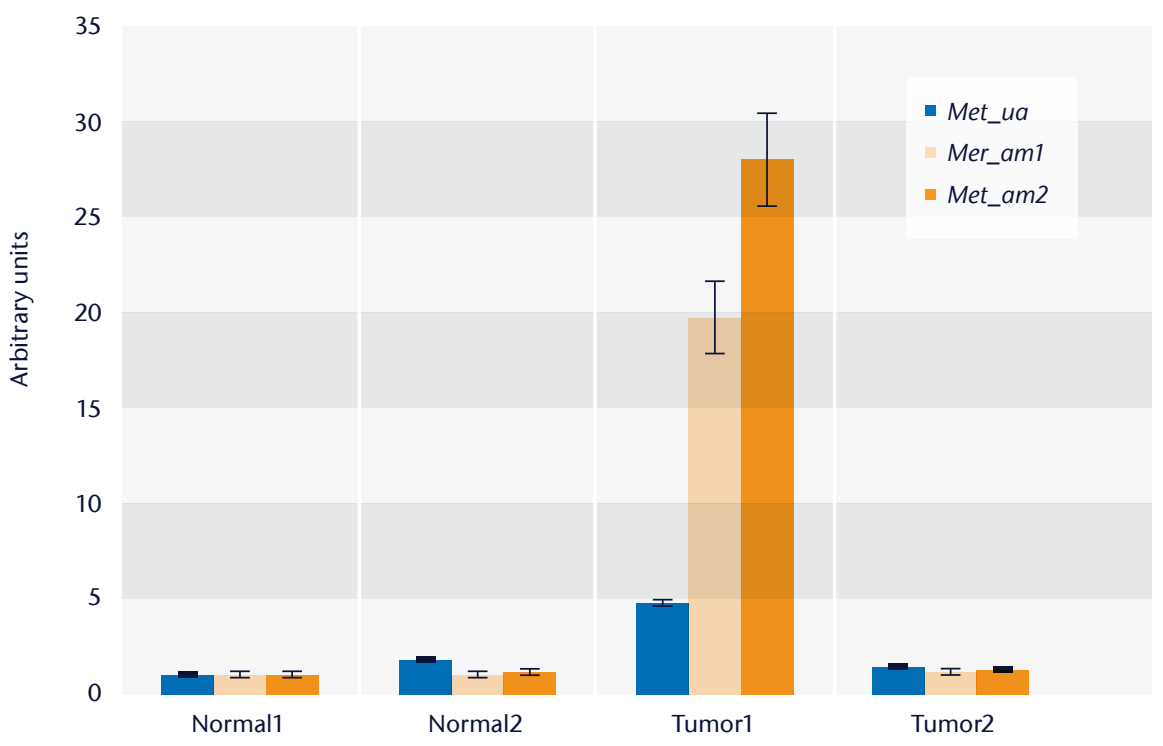

$A b b .4)$ Diese Abbildung zeigt die Ergebnisse der quantitativen $P C R$ zur Verifizierung der errechneten Kopienzahlvariation auf Chromosom 6 (17.4 Mb-18.6 Mb). Beide normalen Proben zeigen keine Erhöhung des Amplikons. Auch im Tumor 2, in dem diese Amplifikation nicht ermittelt werden konnte, ist keine Erhöhung messbar. Lediglich in Tumor 1 wurde eine erhöhte Kopienanzahl gemessen. Dabei liegen die gemessenen Fragmente Met_am1 und Met_am2 in einem Bereich der in Tumor 1 mit erhöhter Kopienanzahl bestimmt wurde, und Met_ua in einem Bereich ohne Kopienzahländerung.

\section{LITERATURVERZEICHNIS}

Hanahan, D., Weinberg, R. A. (2000): The Hallmarks of Cancer. Cell, 100: 57-70.

Klein, A., Guhl, E., Zollinger, R., Tzeng, Y., Wessel, R., Hummel, M., Graessmann, M., Graessmann, A. (2005): Gene expression profiling: cell cycle deregulation and aneuploidy do not cause breast cancer formation in WAPSVT/t transgenic animals. J Mol Med (Berl), 83: 362-376.

Osborne, C., Wilson, P., Tripathyand, D. (2004): Oncogenes and Tumor Suppressor Genes in Breast Cancer: Potential Diagnostic and Therapeutic Applications. Oncologist, 9. 361-377.

Standfuß, C., Pospisil, H., Klein, A. (2012): SNP Microarray analyses reveal copy number alterations and progressive genome reorganization during tumor development in SVT/t driven mice breast cancer. BMC Cancer, 12: 380.

Bergamaschi, A., Kim, Y. H., Wang, P., Sørlie, T., Hernandez-Boussard, T., Lonning, P.E., Tibshirani, R., Børresen-Dale, A., Pollack, J. R. (2006): Distinct patterns of DNA copy number alteration are associated with different clinicopathological features and gene-expression subtypes of breast cancer. Genes Chromosomes Cancer, 45: 1033-1040.

Fanciulli, M., Petretto, E., Aitman, T. (2010): Gene copy number variation and common human disease. Clin Genet, 77: 201-203.

Conrad, D., Pinto, D., Redon, R., Feuk, L., Gokcumen, O., Zhang, Y., Aerts, J., Andrews, T., Barnes, C., Campbell, P., Fitzgerald, T., Hu, M., Ihm, C., Kristiansson, K., Macarthur, D., Macdonald, J., Onyiah, I., Pang, A., Robson, S., Stirrups, K., Valsesia, A., Walter, K., Wei, J., Consortium WTCC, Tyler-Smith, C., Carter, N., Lee, C., Scherer, S., Hurles, M. (2010): Origins and functional impact of copy number variation in the human genome. Nature, 464: 704-712.
Redon, R., Ishikawa, S., Fitch, K., Feuk, L., Perry, G., Andrews, T., Fiegler, H., Shapero, M., Carson, A., Chen, W., Cho, E., Dallaire, S., Freeman, J., Gonz'alez, J., Gratac'os, M., Huang, I., Kalaitzopoulos, D., Komura, D., MacDonald, I., Marshall, C., Mei, R., Montgomery, L., Nishimura, K., Okamura, K., Shen, F., Somerville, M., Tchinda, J., Valsesia, A., Woodwark, C., Yang, F., Zhang, J., Zerjal, T., Zhang, I., Armengol, L., Conrad, D., Estivill, X., Tyler-Smith, C., Carter, N., Aburatani, H., Lee, C., Jones, K., Scherer, S., Hurles, M. (2006): Global variation in copy number in the

Cahan, P., Li, Y., Izumi, M., Graubert, T.A (2009): The impact of copy number variation on local gene expression in mouse hematopoietic stem and progenitor cells. Nat Genet, 14: 430-437.

Henrichsen, C., Vinckenbosch, N., Zöllner, S., Chaignat, E., Pradervand, S., Schütz, F., Ruedi, M., Kaessmann, H., Reymond, A. (2009): Segmental copy number variation shapes tissue transcriptomes. Nat Genet, 41: 424-429.

Hannemann, J., Meyer-Staeckling, S., Kemming, D. Alpers, I., Joosse, S.A., Pospisil, H., Kurtz, S., Görndt, J., Püschel, K., Riethdorf, S., Pantel, K., Brandt, B. (2011): Quantitative High-Resolution Genomic Analysis of Single Cancer Cells. PLoS ONE 2011, 6: e26362.

Jörnsten, R., Abenius, T., Kling, T., Schmidt, L., Johansson, E., Nordling, T., Nordlander, B., Sander, C., Gennemark, P., Funa, K., Nilsson, B., Lindahl, L., Nelander, S. (2011): Network modeling of the transcriptional effects of copy number aberrations in glioblastoma. Mol Syst Biol, 7: 486. human genome. Nature, 444: 444-454.

\section{AUTORENANGABEN}

Christoph Standfuß, MSc.

Bioinformatik

TH Wildau [FH]

Fachbereich Ingenieurwesen/Wirtschaftsingenieurwesen Arbeitsgruppe Bioinformatik/High Performance Computing in Life Sciences

Telefon: +49 (0) 3375 / 508-204

christoph.standfuss@th-wildau.de

Prof. Dr. rer. nat. Heike Pospisi

TH Wildau [FH]

Fachbereich Ingenieurwesen/Wirtschaftsingenieurwesen Arbeitsgruppe Bioinformatik/High Performance Computing in Life Science Telefon: +49 (0) 3375 / 508-949

heike.pospisil@th-wildau.de

\section{Dr. med. Andreas Klein}

Charité - Universitätsmedizin Berlin, Institut für Biochemie Telefon: +49 (0) 30 / 450528087

andreas.klein@charite.de 